\section{MS11-P6 Mechanistic basis for site-specific functions of focal adhesion kinase}

Stefan T. Arold ${ }^{1}$, Karen Brami-Cherrier ${ }^{2}$, Nicolas Gervasi ${ }^{2}$, Katarzyna W. Walkiewicz ${ }^{1}$, Gress Kadareé ${ }^{2}$, Jean-Antoine Girault $^{2}$

1. King Abdullah University of Science and Technology (KAUST), Saudi Arabia

2. Institut du Fer à Moulin, Paris, France

email: stefan.arold@kaust.edu.sa

Focal adhesion kinase (FAK) controls adhesion-dependent cell motility, survival, and proliferation, and plays a major role in development and cancer. Interestingly, FAK has different functions in different cellular compartments. For example at focal adhesions, FAK regulates integrin signalling in a kinase-dependent manner, whereas in the nucleus it exerts kinase-independent anti-apoptotic effects. We have combined SAXS with data from x-ray crystallography, NMR, bioinformatics, biochemical and functional analyses, to provide first structural insights into full-length FAK. Through specifically affecting the structural dynamics of FAK, we show that low-probability conformational transitions are of biological importance. Collectively, our data show how the dynamics and allosteric interplay between ligands and FAK's several domains controls site-specific activity of FAK. Our results reveal how FAK detects the coincidence of multiple signals to generate an environment-specific outcome.

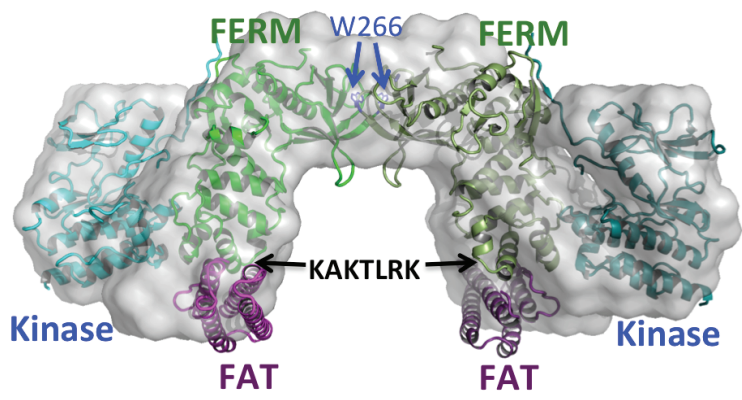

Figure 1. Structure of full-length focal adhesion kinase, obtained by hybrid methods.

Keywords: focal adhesion, kinase, SAXS, NMR, confocal microscopy, x-ray crystallography
MS11-P7 Structural studies of the human Nuclear EXosome Targeting complex

Nicholas Sofos ${ }^{1}$, Mikael B. Winkler ${ }^{1}$, Ditlev E. Brodersen ${ }^{1}$

1. Centre for mRNP Biogenesis and Metabolism, Department of Molecular Biology and Genetics, Aarhus University, DK-8000 Aarhus C

email: sofos@mbg.au.dk

RNA decay is an important process in all domains of life as it is essential for maturation of nuclear RNAs, regulates the abundance of transcripts, and prevents the accumulation of spurious transcripts. The main machinery responsible for 3'-5' decay in eukaryotes is the RNA exosome; a multi-component protein complex consisting of a 9-subunit inactive core and the associated nucleases Rrp44 and Rrp6[1]. In addition, the exosome utilizes a number of different cofactors that stimulate enzymatic activity and serve as adapters for its many substrates. A chief activator of the nuclear exosome is the MTR4 helicase, which mainly functions in the context of the TRAMP complex in human nucleoli, and in the context of the NEXT (Nuclear EXosome Targeting) complex in the nucleoplasm.

The NEXT complex consists of the RNA DexH-box helicase MTR4, the Zn-knuckle protein ZCCHC8, and the putative RNA binding protein RBM7 - with the later two being highly conserved in vertebrates only. Consistent with its residence in the nucleoplasm, NEXT is required for degradation of promoter upstream transcripts (PROMPTs) and other non-coding RNA PolII transcripts[2]'[3].

Individual fragments of the NEXT subunits have been purified for crystallization trials and interaction profiling of core fragments. A fragment of the RBM7 protein has been crystallized and diffraction data has been collected. So far, the data quality is too low and optimization is ongoing.

In a parallel approach, the endogenously expressed NEXT complex has been extracted and purified for negative stain EM. Data processing is ongoing.

[1] Chlebowski, A., Lubas, M., Jensen, T.H., and Dziembowski, A. (2013). RNA decay machines: the exosome. Biochim. Biophys. Acta 1829, 552-560. [2] Lubas, M., Christensen, M.S., Kristiansen, M.S., Domanski, M., Falkenby, L.G., Lykke-Andersen, S., Andersen, J.S., Dziembowski, A., and Jensen, T.H. (2011). Interaction profiling identifies the human nuclear exosome targeting complex. Mol. Cell 43, 624-637. [3] Lubas, M., Andersen, P., Schein, A., Dziembowski, A., Kudla, G., and Jensen, T. (2015). The Human Nuclear Exosome Targeting Complex Is Loaded onto Newly Synthesized RNA to Direct Early Ribonucleolysis. Cell Reports.

Keywords: crystallography, electron microscopy, NEXT complex 